\title{
Reference data for phase diagrams of triangular and hexagonal bosonic lattices
}

\author{
N. Teichmann ${ }^{(a)}$, D. Hinrichs and M. Holthaus \\ Institut für Physik, Carl von Ossietzky Universität, D-26111 Oldenburg, Germany
}

\begin{abstract}
PACS 03.75.Lm - Tunneling, Josephson effect, Bose-Einstein condensates in periodic potentials PACS 64.70. Tg - Quantum phase transitions

PACS $67.85 . \mathrm{Hj}$ - Bose-Einstein condensates in optical potentials
\end{abstract}

\begin{abstract}
We investigate systems of bosonic particles at zero temperature in triangular and hexagonal optical lattice potentials in the framework of the Bose-Hubbard model. Employing the process-chain approach, we obtain accurate values for the boundaries between the Mott insulating phase and the superfluid phase. These results can serve as reference data for both other approximation schemes and upcoming experiments. Since arbitrary integer filling factors $g$ are amenable to our technique, we are able to monitor the behavior of the critical hopping parameters with increasing filling. We also demonstrate that the $g$-dependence of these exact parameters is described almost perfectly by a scaling relation inferred from the mean-field approximation.
\end{abstract}

Introduction. - Over the last ten years ultracold átoms in optical lattices induced by standing waves of laser radiation have become an outstandingly important and intensely studied testing ground for quantum many-body physics [1,2]. Great prospects offered by these systems stem from the chance to investigate condensed-matter phenomena by simulating paradigmatic model Hamiltoniáns in the laboratory 3. In particular, the Bose-Hubbard Hamiltonian [4,5] has attracted a lot of attention, since it describes ultracold bosonic atoms in an optical lattice potential fairly well. This system exhibits a quantum phase transition from a superfluid to a Mott insulator upon increasing the lattice depth 6, 7. Its extensions even show further interesting phases, e.g. a supersolid state [8], when admitting particle-particle interactions between neighboring sites [9] or introducing Bose-Fermi mixtures [10].

So far, most studies dealing with the Bose-Hubbard model have considered a square or a cubic lattice. For these particular lattice geometries the superfluid-insulator phase boundary has been calculated by various methods, such as mean-field approaches [4,11-15], the quantum rotor approach [16], or a variational cluster formulation [17. Arguably, the most precise results have been achieved by the strong coupling expansion [18, 20, and by Quantum Monte Carlo simulations [21,22 for low filling factors of the lattice, and by means of the process-chain approach

(a) E-mail: Teichmann@theorie.physik. uni-oldenburg.de for arbitrarily high integer filling 23 .

Quite recently, the successful experimental realization of planar triangular and hexagonal lattices has been reported 24. However, reliable theoretical data for the phase boundaries pertaining to these lattice types still seem to be missing, except for the single case of a triangular lattice at unit filling $(g=1)$, which has been covered by a strong coupling expansion [25]. Apart from the need to compare experimental results to accurate theoretical predictions, precise knowledge of the critical values of the hopping parameters would also be of great value to aid the development of new approximation schemes, and of future numerical methods.

In this contribution we provide the phase diagrams for the Bose-Hubbard model with planar triangular and hexagonal lattice geometries. These two lattice types are depicted schematically in fig. 1. The process-chain approach [26] in combination with the method of the effective potential [27, 28 enables us to compute the phase boundaries with high precision, as has been demonstrated previously for square and cubic lattices [23, 29].

For self-consistency, we start with a brief description of the Bose-Hubbard model, and give a short explanation of both the process-chain approach and the method of the effective potential, which provides the signature of the phase transition. We then present our results for the phase diagrams arising from triangular and hexagonal lattices, and state the corresponding critical values of the 


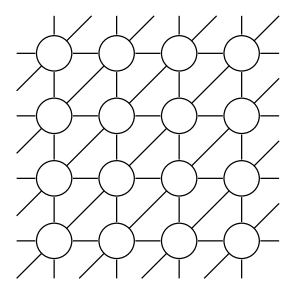

(a)

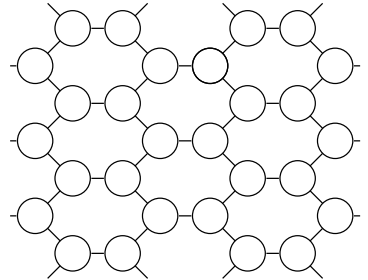

(b)
Fig. 1: Schematic illustration of the triangular (a) and the hexagonal (b) lattice. Circles represent lattice sites occupied by bosonic particles; lines represent nearest-neighbor couplings due to tunneling processes between adjacent sites.

hopping parameter $(J / U)_{c}$ and of the chemical potential $(\mu / U)_{c}$. Since we can treat lattices with an arbitrary number of particles per lattice site, i.e., with an arbitrarily high integer filling factor $g$, we are able to reveal that the critical values $(J / U)_{\mathrm{c}}$ can be scaled such that they become (almost) independent of the filling factor.

The model. - We study the homogeneous BoseHubbard model, given by the Hamiltonian

$$
H=\frac{U}{2} \sum_{i} \hat{n}_{i}\left(\hat{n}_{i}-1\right)-J \sum_{\langle i, j\rangle} \hat{a}_{i}^{\dagger} \hat{a}_{j}-\mu \sum_{i} \hat{n}_{i},
$$

which embodies in an elementary way the competition between the kinetic energy due to tunneling processes and the potential energy associated with the repulsive interaction of bosons on the same lattice site. The operators $\hat{a}_{i}$ and $\hat{a}_{i}^{\dagger}$ are the bosonic annihilation and creation operators at site No. $i$, and $\hat{n}_{i}$ is the corresponding number operator. We examine the case of zero temperature, which permits a single-band description, such that one only needs to consider the lowest Wannier state at each site. Moreover, an on-site approximation is made here, assuming that only particles sitting on the same lattice site interact with each other, each on-site pair contributing the amount $U$ to the total interaction energy. Hopping processes of the bosons are restricted to adjacent sites; their strength is quantified by the matrix element $J$. The subscript $\langle i, j\rangle$ at the kinetic-energy sum indicates that this summation only includes pairs of neighboring sites. For the homogeneous systems studied here, the chemical potential $\mu$ is constant throughout the lattice.

When expressing all energies in multiples of the on-site pair interaction energy $U$, we arrive at the dimensionless Hamiltonian

$$
H_{\mathrm{BH}}=\frac{1}{2} \sum_{i} \hat{n}_{i}\left(\hat{n}_{i}-1\right)-J / U \sum_{\langle i, j\rangle} \hat{a}_{i}^{\dagger} \hat{a}_{j}-\mu / U \sum_{i} \hat{n}_{i}
$$

containing two parameters, the hopping parameter $J / U$ and the scaled chemical potential $\mu / U$.

The existence of a quantum phase transition from a Mott insulator to a superfluid [4,6] in response to an in- crease of the hopping parameter is made plausible by inspecting the limiting cases: When $J / U \gg 1$ one has an almost ideal Bose-Einstein condensate with all particles occupying the zero-quasimomentum Bloch state. This corresponds to a superfluid with all particles delocalized, and phase fluctuations being suppressed. The superfluid phase is characterized by long-range order and non-zero compressibility, $\partial\langle n\rangle / \partial \mu \neq 0$. In the opposite limit $J / U \ll 1$ hopping is prohibited and all sites are decoupled from each other, so that the Hamiltonian (2) becomes diagonal in the occupation number basis. Minimizing the on-site energy, one finds that an integer number $g=N / M$ occupies each site, with $N$ denoting the total number of particles, and $M$ the number of lattice sites. This phase is characterized by reduced density fluctuations and incompressibility, i.e. $\partial\langle n\rangle / \partial \mu=0$. The ground state $|m\rangle$ for $J / U=0$ simply is a product state of Fock states with $g$ particles on each site,

$$
|m\rangle=\prod_{i=1}^{M} \frac{\left(\hat{a}_{i}^{\dagger}\right)^{g}}{\sqrt{g !}}|0\rangle
$$

where $|0\rangle$ is the particle-free vacuum. When starting in the Mott-insulating phase and increasing $J / U$ from zero to higher values for a given, fixed chemical potential $\mu / U$, there is a value $(J / U)_{\mathrm{pb}}$ at which the excitation gap vanishes, marking the entrance into the superfluid regime.

The Method. - In order to determine these values $(J / U)_{\mathrm{pb}}$ of the hopping parameter at the phase boundary we make use of the method of the effective potential 27-29], which requires to add source and drain terms of constant strength $\eta$ and $\eta^{*}$ to the Bose-Hubbard Hamiltonian (2):

$$
\tilde{H}_{\mathrm{BH}}\left(\eta, \eta^{*}\right)=H_{\mathrm{BH}}+\sum_{i}\left(\eta^{*} \hat{a}_{i}+\eta \hat{a}_{i}^{\dagger}\right) .
$$

The corresponding grand canonical free energy

$$
F\left(J / U, \eta, \eta^{*}\right)=M\left(f_{0}(J / U)+\sum_{n=1}^{\infty} c_{2 n}(J / U)|\eta|^{2 n}\right)
$$

with expansion coefficients

$$
c_{2 n}(J / U)=\sum_{\nu=0}^{\infty} \alpha_{2 n}^{(\nu)}(J / U)^{\nu}
$$

then is Legendre-transformed into an effective potential

$$
\Gamma\left(J / U, \psi, \psi^{*}\right)=F_{0}-\frac{1}{c_{2}}|\psi|^{2}+\frac{c_{4}}{c_{2}^{4}}|\psi|^{4}+\mathcal{O}\left(|\psi|^{6}\right) .
$$

Odd orders of $\eta$ vanish in the expansion (5) of the free energy because of the $U(1)$-symmetry of the augmented Hamiltonian (41). The expansion parameter $|\psi|^{2}$ of the effective potential (17) serves as the order parameter; it is given by

$$
\begin{aligned}
\psi(\eta) & =\frac{1}{M} \frac{\partial F}{\partial \eta^{*}}=\left\langle\hat{a}_{i}\right\rangle_{\eta} \\
\psi^{*}(\eta) & =\frac{1}{M} \frac{\partial F}{\partial \eta}=\left\langle\hat{a}_{i}^{\dagger}\right\rangle_{\eta} .
\end{aligned}
$$


The Legendre pair $\eta$ and $\psi^{*}$ obeys the identity

$$
\frac{\partial \Gamma}{\partial \psi^{*}}=-\eta
$$

the complex conjugate of this equation connects $\eta^{*}$ and $\psi$. Now the original Bose-Hubbard Hamiltonian (2) is recovered from the augmented Hamiltonian (4) by setting $\eta=\eta^{*}=0$, which means that the relevant values of $\psi$ and $\psi^{*}$ are those which render the effective potential $\Gamma$ stationary. For low hopping strengths $J / U$, when the system is in its Mott-insulating phase, the coefficient $c_{2}$ in the expansions (6) and (7) is negative, whereas $c_{4}$ is positive, leading to a minimum of $\Gamma$ at $|\psi|^{2}=0$. The order parameter $|\psi|^{2}$ adopts a non-zero value in the superfluid phase, signaling long-range order. The phase transition therefore takes place at that value of $J / U$ for which $1 / c_{2}$ vanishes, so that the minimum of the expression (7) starts to deviate from $|\psi|^{2}=0$. The upshot is that the phase boundary $(J / U)_{\mathrm{pb}}$ equals the radius of convergence of the series (6) for the coefficient $c_{2}$.

The coefficients $\alpha_{2}^{(\nu)}$ of that series are calculated within the process-chain approach, which is based on a diagrammatic evaluation [26,29] of Kato's perturbation series [30]. The Kato formula for the $n$ th-order energy correction experienced by a nondegenerate unperturbed state $|m\rangle$ in response to a perturbation $V$ reads

$$
E_{m}^{(n)}=\operatorname{tr}\left[\sum_{\left\{\alpha_{\ell}\right\}} S^{\alpha_{1}} V S^{\alpha_{2}} V S^{\alpha_{3}} \ldots S^{\alpha_{n}} V S^{\alpha_{n+1}}\right]
$$

Here the sum runs over all sets of $n+1$ non-negative integers $\alpha_{\ell}$ which obey the constraint $\sum_{\ell} \alpha_{\ell}=n-1$. The linking operators $S^{\alpha}$ are defined by

$$
S^{\alpha}=\left\{\begin{array}{cl}
-|m\rangle\langle m| & \text { for } \alpha=0 \\
\sum_{i \neq m} \frac{|i\rangle\langle i|}{\left(E_{m}^{(0)}-E_{i}^{(0)}\right)^{\alpha}} & \text { for } \alpha>0
\end{array},\right.
$$

where $|i\rangle$ denotes the unperturbed "intermediate" eigenstates, and $E_{i}^{(0)}$ the corresponding unperturbed eigenvalues. Kato's trace formula (10) can be rewritten as a sum of matrix elements of the state $|m\rangle$ considered,

$$
\left\langle m\left|V S^{\alpha_{1}} V S^{\alpha_{2}} \ldots S^{\alpha_{n-1}} V\right| m\right\rangle .
$$

The number of such matrix elements (Kato-terms) quickly increases with the order $n$ of perturbation theory. In first order, the only Kato-term is $\langle m|V| m\rangle$, while $n=2$ leads to $\left\langle m\left|V S^{1} V\right| m\right\rangle$. These are precisely the well known firstand second- order energy corrections, as becomes obvious when inserting $S^{1}$ from eq. (11). Each Kato-term (12) can be viewed as a (sum of) closed process chain(s) consisting of $n$ processes caused by the perturbation $V$, leading from the state $|m\rangle$ over various intermediate states $|i\rangle$ back to $|m\rangle$ again. When dealing with a homogeneous lattice system, many process chains can be combined into diagrams

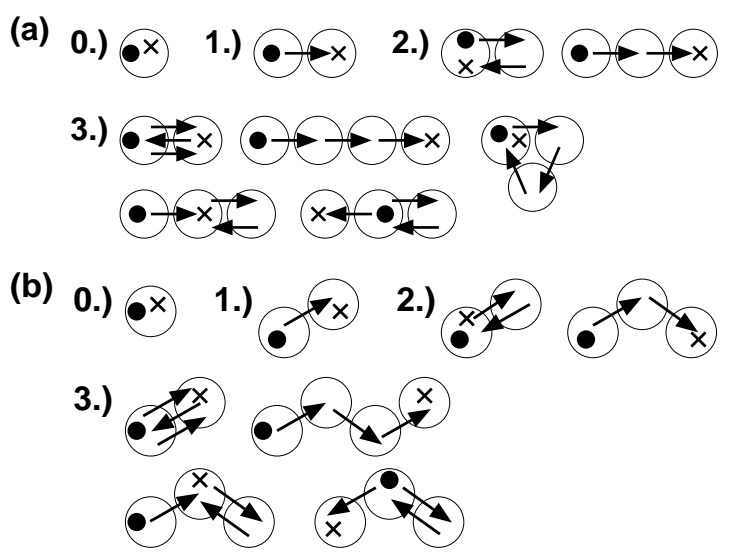

Fig. 2: Diagrams of order $\nu=0, \ldots, 3$ in the hopping parameter $J / U$ for a triangular and for a hexagonal lattice. The symbol $\bullet$ indicates the creation of a particle, $\rightarrow$ corresponds to tunneling between two adjacent sites, and $\times$ to an annihilation process. Upper figure (a): Diagrams for the triangular lattice; weight factors are 0.) 1 ; 1.) 6 ; 2.) 6 and 30 ; 3.) $6,138,12,30$ and 30. Lower figure (b): Diagrams for the hexagonal lattice; weight factors are 0.) 1 ; 1.) 3 ; 2.) 3 and 6 ; 3.) $3,12,6$ and 6 .

by appending an appropriate weight factor. This procedure drastically reduces the numerical effort. A more detailed description of the application of this process-chain technique to the Bose-Hubbard model is given in ref. [29].

In our case, the unperturbed part of the Hamiltonian is site-diagonal, reading

$$
H_{0}=\frac{1}{2} \sum_{i} \hat{n}_{i}\left(\hat{n}_{i}-1\right)-\mu / U \sum_{i} \hat{n}_{i} .
$$

The perturbation is given by the tunneling operators in combination with the source and drain terms artificially introduced in eq. (4):

$$
V=-J / U \sum_{\langle i, j\rangle} \hat{a}_{i}^{\dagger} \hat{a}_{j}+\sum_{i}\left(\eta^{*} \hat{a}_{i}+\eta \hat{a}_{i}^{\dagger}\right) .
$$

Instead of using Kato's formulation for computing the total energy corrections, we employ it for calculating the coefficients $\alpha_{2}^{(\nu)}$ of the series (6) for $c_{2}$ only; the searched-for phase boundary $(J / U)_{\mathrm{pb}}$ then is determined in a second step as the radius of convergence of this series. Because $c_{2}$ is the coefficient of $|\eta|^{2}$ in the expansion of the free energy (5), it is associated with exactly one creation and one annihilation event of a particle. Hence, for calculating its coefficients $\alpha_{2}^{(\nu)}$ one has to evaluate only diagrams containing one creation (symbolized by a dot: •) and one annihilation process $(\times)$, together with $\nu$ tunneling processes $(\rightarrow)$.

Both the precise structure of the diagrams and their weight factors are determined by the geometry of the underlying lattice. Figure 2 lists the diagrams of order $\nu=0, \ldots, 3$ in the hopping parameter $J / U$ for a triangular and for a hexagonal lattice, together with their respective weight factors. Each "hexagonal" diagram shown in 


\begin{tabular}{c|r|r|r|r|r|r|r|r|r|r|r}
\hline$\nu$ & 0 & 1 & 2 & 3 & 4 & 5 & 6 & 7 & 8 & 9 & 10 \\
\hline Triangular & 1 & 1 & 2 & 5 & 14 & 41 & 129 & 416 & 1398 & & \\
Hexagonal & 1 & 1 & 2 & 4 & 9 & 18 & 39 & 80 & 180 & 389 & 1260 \\
\hline
\end{tabular}

Table 1: Number of diagrams to be evaluated when calculating the phase boundary of the Bose-Hubbard model for a triangular and for a hexagonal lattice to $\nu$ th order in the hopping parameter $J / U$, corresponding to the order $\nu+2$ of Kato's perturbation series.

(b) is topologically equivalent to a "triangular" one in (a), but when taking three hopping processes into account a circular diagram turns up in the triangular case which has no hexagonal counterpart. In higher orders of the hopping parameter the number of "triangular" diagrams even becomes much larger than that of the "hexagonal" ones, as table 1 documents: The increase of the number of diagrams with the number $\nu$ of tunneling processes is much more pronounced in the triangular case. As another consequence of the geometric variation, the weight factors of corresponding diagrams generally differ for the two lattice types.

The numerical value of a diagram is determined by going through all permutations of its individual constituent processes; for each permutation one has to evaluate those Kato-terms which match it. The outcome then is multiplied by the weight factor of the diagram in question. Finally the contributions of all diagrams occurring in a given order of perturbation theory are summed to yield the desired quantity $a_{2}^{(\nu)}$. For example, when considering the hexagonal lattice with $\nu=3$ tunneling processes, four diagrams depicted in fig. 2(b) have to be dealt with. Each one of these leads to up to $5 !=120$ different sequences of processes which have to be matched with 3 Kato-terms. Evidently the computational effort increases rapidly with the number $\nu$ of tunneling processes taken into account: Both the number of Kato-terms and the number of diagrams proliferates quickly; in addition, the number of process permutations grows factorially with the order $n=\nu+2$ of perturbation theory.

Results. - For each preselected value of the chemical potential $\mu / U$, the corresponding coefficients $\alpha_{2}^{(\nu)}$ of the series (6) for $c_{2}$ show an almost geometric behavior, for both the triangular and the hexagonal lattice. As outlined above, the boundary $(J / U)_{\mathrm{pb}}$ between the Mott insulating and the superfluid phase is given by the lowest $J / U$ for which this series diverges. Thus, for delineating the phase boundary we determine its radius of convergence by means of d'Alembert's ratio test 31:

$$
(J / U)_{\mathrm{pb}}=\lim _{\nu \rightarrow \infty}\left|\frac{\alpha_{2}^{(\nu-1)}}{\alpha_{2}^{(\nu)}}\right| .
$$

The required extrapolation $\nu \rightarrow \infty$ is carried out by a linear fit of the ratios $\alpha_{2}^{(\nu-1)} / \alpha_{2}^{(\nu)}$ over $1 / \nu$; the desired value $(J / U)_{\mathrm{pb}}$ then is the point of intersection with the
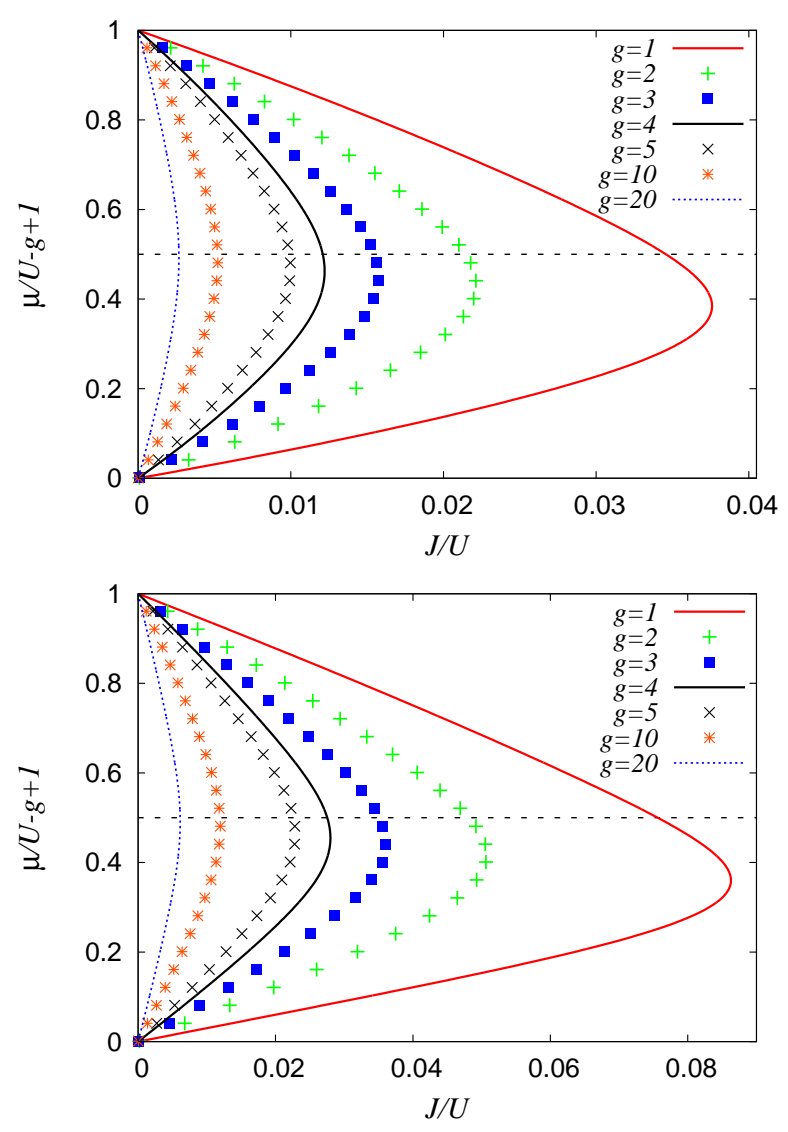

Fig. 3: (Color online) Mott lobes for the triangular lattice (upper panel) and for the hexagonal lattice (below) for various filling factors $g$. The dashed horizontal line $\mu / U=g-0.5$ marks the axis of particle-hole symmetry which appears in the limit of large $g$.

ordinate. This procedure also gives access to the relative error of $(J / U)_{\mathrm{pb}}$ : Varying the set of coefficients $\alpha_{2}^{(\nu)}$ employed for the fit (e.g., taking only $\nu=4, \ldots, 8)$ yields slightly different results; such fluctuations quantify the uncertainty of the final data. Here we employ the coefficients $\nu=2, \ldots, 8$, leading to an estimated relative error of less than $1 \%$ for the triangular case, and about $2 \%$ for the hexagonal one.

The phase diagrams for the two lattice types are plotted in fig. 3 in the $\mu / U$ vs. $J / U$-plane, for various filling factors $g$. The critical values $(\mu / U)_{\mathrm{c}}$ and $(J / U)_{\mathrm{c}}$, i.e. the chemical potential and the hopping parameter at the tip of the respective Mott lobe, are listed in table 2. Our result for the triangular lattice with unit filling compares favorably to the previous finding of Elstner and Monien [25]: These authors have stated $(J / U)_{\mathrm{c}}=0.037785$, whereas we obtain $(J / U)_{\mathrm{c}}=0.03759$; the deviation of about $0.5 \%$ is well within the estimated error margin. Because the coordination number $z_{\mathrm{t}}=6$ of the triangular lattice is twice as large as that for the hexagonal one, $z_{\mathrm{h}}=3$, the "triangular" critical hopping strength at unit filling is substantially lower - by a factor of about 2.3 - than the "hexagonal" 


\begin{tabular}{r|r|r|r|r}
\hline & \multicolumn{2}{|c|}{ Triangular } & \multicolumn{2}{c}{ Hexagonal } \\
\hline$g$ & $(\mu / U)_{\mathrm{c}}$ & $(J / U)_{\mathrm{c}}$ & $(\mu / U)_{\mathrm{c}}$ & $(J / U)_{\mathrm{c}}$ \\
\hline 1 & 0.384 & $3.759 \mathrm{E}-02$ & 0.360 & $8.628 \mathrm{E}-02$ \\
2 & 1.432 & $2.214 \mathrm{E}-02$ & 1.418 & $5.075 \mathrm{E}-02$ \\
3 & 2.452 & $1.574 \mathrm{E}-02$ & 2.442 & $3.606 \mathrm{E}-02$ \\
4 & 3.463 & $1.222 \mathrm{E}-02$ & 3.455 & $2.799 \mathrm{E}-02$ \\
5 & 4.469 & $9.984 \mathrm{E}-03$ & 4.463 & $2.288 \mathrm{E}-02$ \\
10 & 9.484 & $5.222 \mathrm{E}-03$ & 9.481 & $1.196 \mathrm{E}-02$ \\
20 & 19.492 & $2.674 \mathrm{E}-03$ & 19.490 & $6.125 \mathrm{E}-03$ \\
40 & 39.496 & $1.353 \mathrm{E}-03$ & 39.495 & $3.100 \mathrm{E}-03$ \\
50 & 49.497 & $1.085 \mathrm{E}-03$ & 49.496 & $2.486 \mathrm{E}-03$ \\
100 & 99.498 & $5.453 \mathrm{E}-04$ & 99.498 & $1.249 \mathrm{E}-03$ \\
1000 & 999.500 & $5.477 \mathrm{E}-05$ & 999.500 & $1.255 \mathrm{E}-04$ \\
10000 & 9999.500 & $5.480 \mathrm{E}-06$ & 9999.500 & $1.255 \mathrm{E}-05$ \\
\hline
\end{tabular}

Table 2: Critical values $(\mu / U)_{\mathrm{c}}$ and $(J / U)_{\mathrm{c}}$ for various filling factors $g$. For locating the tip of the respective Mott lobe, $\mu / U$ has been varied in steps of 0.001 . Relative errors of $(J / U)$ c are less than $1 \%$ in the triangular case, and about $2 \%$ for the hexagonal lattice.

one. On the other hand, despite the fact that the coordination number of the triangular lattice coincides with that of the simple three-dimensional (3D) cubic lattice, the corresponding critical hopping strengths differ appreciably: The cubic lattice yields $(J / U)_{\mathrm{c}} \approx 0.0341$ for $g=1$, see refs. [22, 23, amounting to a deviation of approximately $9 \%$ from the triangular-lattice value. Inspecting the Mott lobes in fig. 3, one also confirms that the critical chemical potential $(\mu / U)_{\mathrm{c}}$ tends to $g-0.5$ with increasing filling factor $g$, as expected from the particle-hole symmetry which emerges in the large- $g$-limit.

Figure 3 also illustrates that the critical values $(J / U)_{\mathrm{c}}$ decrease with increasing filling factor $g$. As we have shown previously 32, in the cases of the $2 \mathrm{D}$ square and the $3 \mathrm{D}$ cubic lattices the $g$-dependence of the exact critical values is quite well captured by the mean-field expression [4] for $(J / U)_{\mathrm{c}}$, even though the numerical agreement of the mean-field solution with the exact data is only moderate. Thus, the scaled critical values

$$
(J / U)_{\mathrm{c}}^{\mathrm{sc}}=\sqrt{g(g+1)}\left[\frac{1}{2}+\sqrt{\frac{1}{4}+\frac{1}{16 g(g+1)}}\right](J / U)_{\mathrm{c}}
$$

are almost independent of $g$. Here we demonstrate that this finding also applies to the triangular and to the hexagonal lattice by plotting in fig. 4 the scaled data for both cases. As testified by the rather fine scale of the ordinate these scaled data are practically constant, with their residual variation amounting to only about $0.1 \%$, which is an order of magnitude smaller than the estimated relative error committed in our present process-chain calculation. Finally, fig. 5 shows the triangular-lattice Mott lobes after applying the scaling (16) not only to $(J / U)_{\mathrm{c}}$, but to the entire phase boundaries. The scaled boundaries associated with different filling factors are quite similar; the remain-
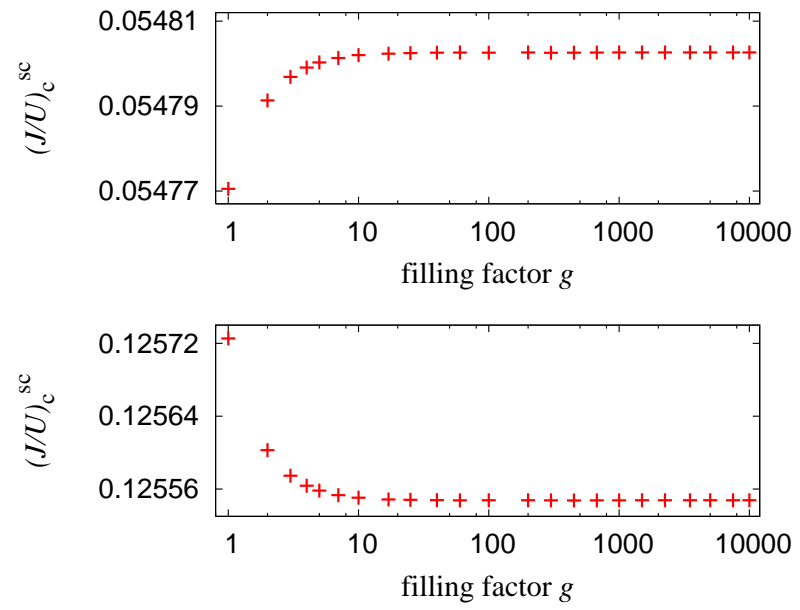

Fig. 4: (Color online) Scaled critical values $(J / U)_{\mathrm{c}}^{\mathrm{sc}}$ according to eq. (16) for the triangular (upper plot) and for the hexagonal lattice (lower plot) vs. the filling factor $g$. Note the very fine scale of the ordinate.

ing differences can be traced mainly to the particle-hole asymmetry of the Bose-Hubbard Hamiltonian. Naturally, this asymmetry is reduced with increasing $g$.

Conclusion. - We have presented fairly accurate phase boundaries for the homogeneous Bose-Hubbard model at zero temperature on both a triangular and on a hexagonal planar lattice, for filling factors ranging from unity to values so high that particle-hole symmetry is practically restored. The calculation has made use of the process-chain approach 26, which already had proven its high fidelity for simple cubic lattices [23, 29]. Our numerical results can serve as benchmark data for other theoretical approaches, and guide upcoming experiments with ultracold atoms in triangular and hexagonal optical lattices 24]. Furthermore, we have shown that the mean-field scaling (16) of the critical values $(J / U)_{\mathrm{c}}$ renders these data almost independent of the filling factor for both lattice types considered here. This $g$-independence of the data scaled in this manner thus appears to be a general feature of the Bose-Hubbard model, without being restricted to particular lattice geometries, while the lattice-specific scaled values $(J / U)_{\mathrm{c}}^{\mathrm{sc}}$ themselves may warrant further deliberations.

$$
* * *
$$

N.T. wishes to thank T.P. Polak for stimulating discussions. Moreover, financial support by the Deutsche Forschungsgemeinschaft (DFG) under grant No. HO $1771 / 5$ is gratefully acknowledged. Computational ressources have been provided by the GOLEM I cluster of the Universität Oldenburg. 


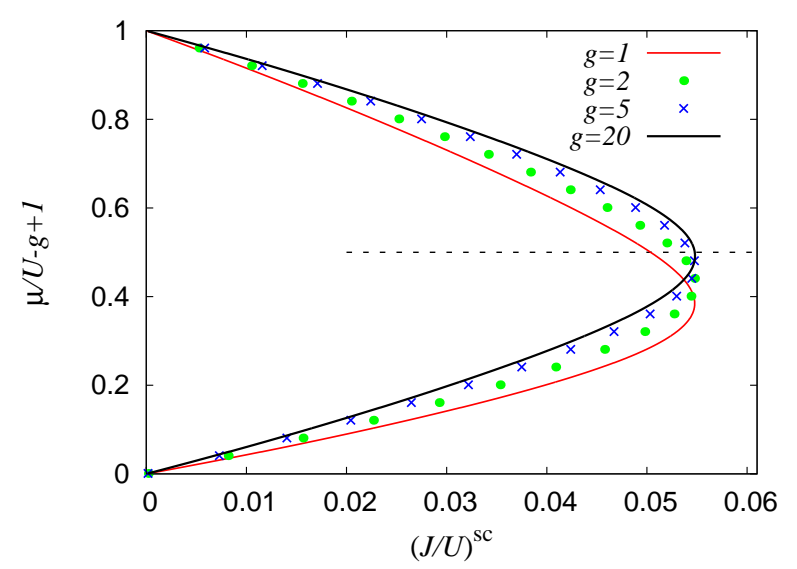

Fig. 5: (Color online) Scaled phase diagrams for the triangular lattice with various filling factors $g$. The scaling provided by eq. (16) stretches the different Mott lobes such that their tips fall at the common value $(J / U)_{\mathrm{c}}^{\mathrm{sc}}$. The remaining differences are caused by the particle-hole asymmetry of the Bose-Hubbard Hamiltonian. Again, the dashed horizontal line $\mu / U=g-0.5$ marks the axis of symmetry which shows up for sufficiently large $g$.

\section{REFERENCES}

[1] Lewenstein M., Sanpera A., Ahufinger V., Damski B., Sen A. and Sen U., Adv. Phys., 56 (2007) 243.

[2] Bloch I., Dalibard J. and Zwerger W., Rev. Mod. Phys., 80 (2008) 885.

[3] Buluta I. and Nori F., Science, 326 (2009) 108.

[4] Fisher M. P. A., Weichman P. B., Grinstein G. and Fisher D. S., Phys. Rev. B, 40 (1989) 546.

[5] Jaksch D., Bruder C., Cirac J. I., Gardiner C. W. and Zoller P., Phys. Rev. Lett., 81 (1998) 3108.

[6] Greiner M., Mandel O., Esslinger T., Hänsch T. W. and Bloch I., Nature, 415 (2002) 39.

[7] Zwerger W., J. Opt. B, 5 (2003) S9.

[8] Leggett A. J., Phys. Rev. Lett., 25 (1970) 1543.

[9] Scarola V. W., Demler E. and Das Sarma S., Phys. Rev. A, 73 (2006) 051601(R).

[10] Titvinidze I., Snoek M. and Hofstetter W., Phys. Rev. Lett., 100 (2008) 100401.

[11] Kampf A. P. and Zimanyi G. T., Phys. Rev. B, 47 (1993) 279.

[12] Bruder C., Fazio R. and Schön G., Phys. Rev. B, 47 (1993) 342.

[13] van Oosten D., van der Straten P. and Stoof H. T. C., Phys. Rev. A, 63 (2001) 053601.

[14] van Oosten D., van der Straten P. and Stoof H. T. C., Phys. Rev. A, 67 (2003) 033606.

[15] Schroll C., Marquardt F. and Bruder C., Phys. Rev. A, 70 (2004) 053609.

[16] Polak T. P. and Kopeć T. K., Phys. Rev. B, 76 (2007) 094503.

[17] Knap M., Arrigoni E. and von der Linden W., Phys. Rev. B, 81 (2010) 024301.

[18] Freericks J. K. and Monien H., Phys. Rev. B, 53 (1996) 2691.

[19] Elstner N. and Monien H., Phys. Rev. B, 59 (1999)
12184.

[20] Freericks J. K., Krishnamurthy H. R., Kato Y., Kawashima N. and Trivedi N., Phys. Rev. A, 79 (2009) 053631.

[21] Capogrosso-Sansone B., Prokof'ev N. V. and Svistunov B. V., Phys. Rev. B, 75 (2007) 134302.

[22] Capogrosso-Sansone B., Ş.G. Söyler, Prokof'ev N. and Svistunov B., Phys. Rev. A, 77 (2008) 15602.

[23] Teichmann N., Hinrichs D., Holthaus M. and Eckardt A., Phys. Rev. B, 79 (2009) 100503(R).

[24] Becker C., Soltan-Panahi P., Kronjäger J., Dörscher S., Bongs K. and Sengstock K., arXiv:0912.3646, (2009) .

[25] Elstner N. and Monien H., arXiv:cond-mat/9905367, (1999) .

[26] Eckardt A., Phys. Rev. B, 79 (2009) 195131.

[27] Negele J. W. and Orland H., Quantum many-particle systems (Westview Press, Reading, MA) 1998.

[28] Dos Santos F. E. A. and Pelster A., Phys. Rev. A, 79 (2009) 013614.

[29] Teichmann N., Hinrichs D., Holthaus M. and Eckardt A., Phys. Rev. B, 79 (2009) 224515.

[30] Kato T., Prog. Theor. Phys., 4 (1949) 514.

[31] Whittaker E. T. and Watson G. N., A course of modern analysis (Cambridge University Press, Cambridge) 2000.

[32] Teichmann N. and Hinrichs D., Eur. Phys. J. B, 71 (2009) 219. 